\title{
Orbital periods and component masses of three double white dwarfs
}

\author{
A. Rebassa-Mansergas ${ }^{1,2}$, S.G. Parsons ${ }^{3}$, E. García-Berro ${ }^{1,2}$, B.T. Gänsicke ${ }^{4}$, \\ M.R. Schreiber ${ }^{5,6}$, M. Rybicka ${ }^{7}$, D. Koester ${ }^{8}$ \\ 1 Departament de Física, Universitat Politècnica de Catalunya, c/Esteve Terrades 5, 08860 Castelldefels, Spain \\ 2 Institute for Space Studies of Catalonia, c/Gran Capità 2-4, Edif. Nexus 201, 08034 Barcelona, Spain \\ 3 Deparment of Physics $\&$ Astronomy, University of Sheffield, Sheffield S3 7RH, UK \\ 4 Department of Physics, University of Warwick, Coventry CV4 7AL, UK \\ 5 Instituto de Física y Astronomía, Universidad de Valparaíso, Avenida Gran Bretaña 1111, Valparaíso, Chile \\ 6 Millenium Nucleus "Protoplanetary Disks in ALMA Early Science", Universidad de Valparaíso, Avenida Gran Bretaña 1111, Valparaíso, Chile \\ 7 Copernicus Astronomical Center, Warszawa, Poland \\ 8 Institut für Theoretische Physik und Astrophysik, University of Kiel, 24098 Kiel, Germany
}

Accepted 2016. Received 2016; in original form 2016

\begin{abstract}
The merger of close double white dwarfs (CDWDs) is one of the favourite evolutionary channels for producing Type Ia supernovae (SN Ia). Unfortunately, current theories of the evolution and formation of CDWDs are still poorly constrained and have several serious uncertainties, which affect the predicted SN Ia rates. Moreover, current observational constraints on this evolutionary pathway for SN Ia mainly rely on only 18 double-lined and/or eclipsing CDWDs with measured orbital and stellar parameters for both white dwarfs. In this paper we present the orbital periods and the individual masses of three new double-lined CDWDs, derived using a new method. This method employs mass ratios, the $\mathrm{H} \alpha$ core ratios and spectral model-fitting to constrain the masses of the components of the pair. The three CDWDs are WD0028-474 $\left(P_{\text {orb }}=9.350 \pm 0.007\right.$ hours, $\left.M_{1}=0.60 \pm 0.06 \mathrm{M}_{\odot}, M_{2}=0.45 \pm 0.04 \mathrm{M}_{\odot}\right), \mathrm{HE} 0410-$ $1137\left(P_{\text {orb }}=12.208 \pm 0.008\right.$ hours, $\left.M_{1}=0.51 \pm 0.04 \mathrm{M}_{\odot}, M_{2}=0.39 \pm 0.03 \mathrm{M}_{\odot}\right)$ and SDSSJ031813.25-010711.7 $\left(P_{\text {orb }}=45.908 \pm 0.006\right.$ hours, among the longest period systems, $\left.M_{1}=0.40 \pm 0.05 \mathrm{M}_{\odot}, M_{2}=0.49 \pm 0.05 \mathrm{M}_{\odot}\right)$. While the three systems studied here will merge in timescales longer than the Hubble time and are expected to become single massive $\left(\gtrsim 0.9 \mathrm{M}_{\odot}\right)$ white dwarfs rather than exploding as SN Ia, increasing the small sample of CDWDs with determined stellar parameters is crucial for a better overall understanding of their evolution.
\end{abstract}

Key words: (stars:) white dwarfs; (stars:) binaries: spectroscopic

\section{INTRODUCTION}

Close double white dwarfs (CDWDs) are close compact binary stars composed of two white dwarfs. CDWDs are of outstanding importance in the general astrophysical context. First, they are the most common type of close compact binary stars in the Galaxy and since their orbital separations continuously decrease through the emission of gravitational waves, they are likely to determine the background noise level of future space-based gravitational wave interferometers, such as LISA (Hils et al. 1990; Ruiter et al. 2010; Marsh 2011). In addition, angular momentum loss through the emission of gravitational waves eventually leads to the merger of the two white dwarfs. If the resulting mass of the merger is $\gtrsim 1.4 \mathrm{M}_{\odot}$, then this event is expected to lead to a Type Ia supernoave explosion (Di Stefano 2010; Toonen et al. 2012; Rebassa-Mansergas et al. 2015). However, currently we do not fully understand how CDWDs form. Thus, predicting the merger rates of CDWDs, or understanding their parameter distributions, or assessing the number of gravitational wave sources are problems that are still affected by serious uncertainties.

The standard scenario for the formation of CDWDs predicts that they are the descendants of main sequence binaries that evolve through two common envelope (CE) episodes (Webbink 2008). The first CE episode occurs when the initially more massive main sequence star evolves into a red giant and overfills its Roche-lobe. Dynamically unstable mass transfer from the giant onto the main sequence companion makes it to also fill its Roche lobe. Thus, both the core of 
the giant and the main sequence companion orbit inside an envelope that is composed mainly by the outer layers of the giant star. Within the $\mathrm{CE}$, drag forces lead to a significant shrinkage of the orbit and the release of orbital energy eventually ejects the envelope, leaving behind a post-CE binary containing a white dwarf and a main sequence companion. The second CE phase begins when the latter evolves into a red giant, producing a CDWD with a typical orbital period of hours to days.

Even though the standard formation scenario of CDWDs was proposed about three decades ago (Webbink 1984), population synthesis models of CDWDs are still far from being able to reproduce essential characteristics of the observed population (e.g. Nelemans et al. 2000; Nelemans \& Tout 2005; Toonen et al. 2012). This is mostly because the CE phase involves a large number of hydrodynamic and thermodynamic processes acting over very wide ranges in time and length scales. Consequently the $\mathrm{CE}$ is commonly described by simple parametrized models (Iben \& Livio 1993; Zuo \& Li 2014). To make things worse, it recently turned out to be unclear whether CDWDs are formed through two CE episodes or by one process of stable conservative mass transfer followed by a CE phase (Woods et al. 2012).

Observationally, recent surveys such as the Sloan Digital Sky Survey (SDSS) or the SN Ia Progenitor SurveY (SPY) have allowed the identification of large numbers of CDWDs that have been used to constrain SN Ia formation channels (Napiwotzki et al. 2007; Badenes \& Maoz 2012; Maoz \& Hallakoun 2016). However, these studies rely on Monte Carlo simulations aimed at reproducing the observational data, which suffer from the uncertainties above outlined. Hence, the only way forward is to directly measure the orbital periods and two component masses of a large sample of CDWDs, which allows obtaining direct constraints on their past evolution, and to thus provide the much needed observational input to test the theoretical models (Nelemans \& Tout 2005; van der Sluys et al. 2006; Woods et al. 2012). This is only possible when analysing double-lined CDWDs, which allows measuring the semiamplitude velocities of the two components and hence provide a direct measure of the mass ratio (e.g. Moran et al. 1997; Napiwotzki et al. 2002), or eclipsing systems, which allow measuring the component masses through the analysis of the observed light curves (e.g. Steinfadt et al. 2010; Parsons et al. 2011).

During the last few years 18 of such CDWDs with measured orbital periods and components masses have been identified (see Table 1). In this paper we derive the orbital periods and component masses of three additional CDWDs (SDSSJ031813.25-010711.7, HE0410-1137 and WD0028474 ), thus increasing the number of CDWDs with measured parameters by $\sim 20$ per cent.

\section{OBSERVATIONS}

We observed SDSSJ031813.25-010711.7 (hereafter SDSSJ0318-0107) as part of a radial velocity survey dedicated to identify CDWDs among apparently single white dwarfs from the Sloan Digital Sky Survey (RebassaMansergas et al., in preparation). SDSSJ0318-0107 turned
Table 1. Orbital periods and component masses of the 18 previously known double-lined and/or eclipsing CDWDs. The masses should be considered as best possible values when no errors are provided. This table supersedes Table 1 of Marsh (2011).

\begin{tabular}{ccccccc}
\hline \hline Object & $\begin{array}{c}M_{1} \\
\left(\mathrm{M}_{\odot}\right)\end{array}$ & $\begin{array}{c}\text { Error } \\
\left(\mathrm{M}_{\odot}\right)\end{array}$ & $\begin{array}{c}M_{2} \\
\left(\mathrm{M}_{\odot}\right)\end{array}$ & $\begin{array}{c}\text { Error } \\
\left(\mathrm{M}_{\odot}\right)\end{array}$ & $\begin{array}{c}P_{\text {orb }} \\
\text { hours })\end{array}$ & Reference \\
\hline WD0135-052 & 0.47 & - & 0.52 & - & 37.35 & $(1)(2)$ \\
PG1101+364 & 0.36 & - & 0.31 & - & 3.47 & $(3)(2)$ \\
WD0957-666 & 0.37 & 0.02 & 0.32 & 0.03 & 1.46 & $(4)(2)$ \\
WD1704+481 & 0.39 & 0.05 & 0.56 & 0.07 & 3.48 & $(5)(2)(*)$ \\
PG1115+166 & 0.70 & - & 0.70 & - & 722.2 & $(6)(7)(\mathrm{x})$ \\
WD0136+768 & 0.47 & - & 0.37 & - & 33.77 & $(2)$ \\
WD1204+450 & 0.46 & - & 0.52 & - & 38.47 & $(2)$ \\
HE1414-0848 & 0.71 & - & 0.55 & - & 12.43 & $(8)$ \\
HE2209-1444 & 0.58 & - & 0.58 & - & 6.65 & $(9)$ \\
WD1349+144 & 0.44 & - & 0.44 & - & 53.02 & $(10)$ \\
NLTT 11748 & 0.15 & 0.05 & 0.71 & 0.06 & 5.66 & $(11)(12)(+)$ \\
CSS 41177 & 0.38 & 0.02 & 0.32 & 0.01 & 2.78 & $(13)(14)(+)$ \\
SDSSJ0651+2844 & 0.55 & - & 0.25 & - & 0.20 & $(15)(+)$ \\
SDSSJ0106-1003 & 0.43 & - & 0.17 & - & 0.65 & $(16)$ \\
SDSSJ1257+5428 & 1.00 & - & 0.20 & - & 4.56 & $(17)$ \\
GALEXJ1717+6757 & 0.90 & - & 0.18 & - & 5.91 & $(18)(+)$ \\
SDSSJ0751-0141 & 0.97 & 0.06 & 0.19 & 0.02 & 1.90 & $(19)(+)$ \\
SDSSJ1152+0248 & 0.44 & 0.09 & 0.41 & 0.11 & 2.39 & $(20)(+)$ \\
\hline 1) & & & & & &
\end{tabular}

(1) Saffer et al. (1988); (2) Maxted et al. (2002b); (3) Marsh (1995); (4) Moran et al. (1997); (5) Maxted et al. (2000); (6) Maxted et al. (2002a); (7) Bergeron \& Liebert (2002); (8) Napiwotzki et al. (2002); (9) Karl et al. (2003b); (10) Karl et al. (2003a); (11) Steinfadt et al. (2010); (12) Kaplan et al. (2014); (13) Parsons et al. (2011); (14) Bours et al. (2014); (15) Brown et al. (2011); (16) Kilic et al. (2011); (17) Marsh et al. (2011); (18) Vennes et al. (2011); (19) Kilic et al. (2014); (20) Hallakoun et al. (2016); (*)Triple system; (x) DB+DA binary; (+) Eclipsing binary.

out to be a double-lined binary. We therefore targeted this system for intense follow-up spectroscopy. In addition, we obtained follow-up spectroscopy of HE0410-1137 and WD0028-474, two additional double-lined CDWDs identified by Koester et al. (2009) with no orbital periods measured.

We performed the observations using the Gemini South telescope in Cerro Pachón and the Magellan Clay telescope in Cerro Las Campanas, both in Chile. We also found Very Large Telescope (VLT) UVES data in the ESO (European Southern Observatory) archive (PI R. Napiwotzki) that we added to our own data. We used the molly package ${ }^{1}$ to apply the heliocentric correction to all spectra. We did not perform flux calibration to our data.

The Gemini South telescope was equipped with the GMOS spectrograph and the B1200 grating. The central wavelength was $597 \mathrm{~nm}$ and the slit width 1 ". We binned the CCD $2 \times 2$. This resulted in spectra of a resolving power of 7000 covering the $\sim 520-675 \mathrm{~nm}$ wavelength range. We reduced and calibrated the data using the pamela ${ }^{2}$ and molly packages, respectively. The observations were performed in service mode during 2013.

The Magellan Clay telescope was equipped with the

1 http://deneb.astro.warwick.ac.uk/phsaap/software/molly/html/INDEX.htm 2 Pamela is distributed as part of The Starlink Project. 


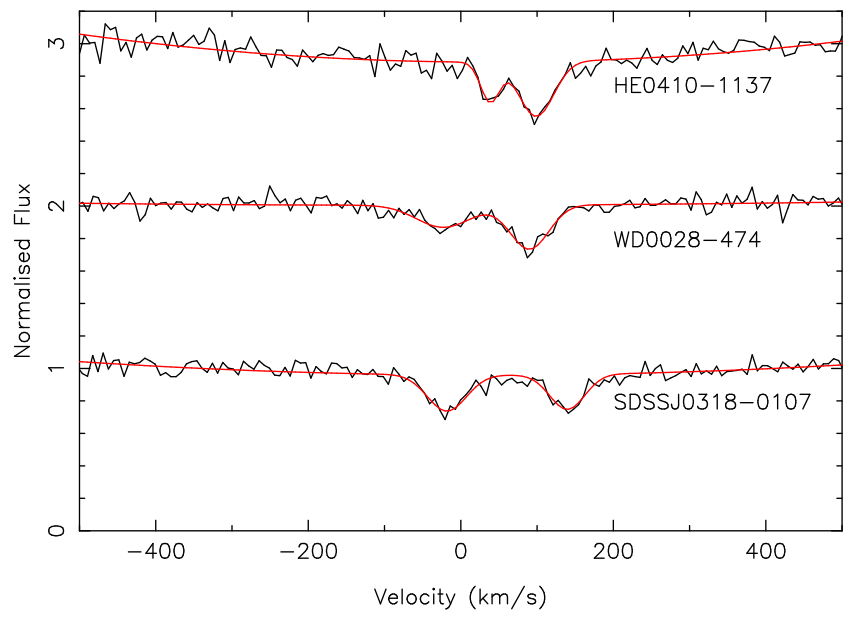

Figure 1. Example Magellan Clay/MIKE spectra of the $\mathrm{H} \alpha$ line cores of our three systems taken at phases where both components are visible. Over-plotted in red are Gaussian fits.

MIKE double echelle spectrograph, which provides spectra in the full optical range $(320-500 \mathrm{~nm}$ in the blue and 490$1000 \mathrm{~nm}$ in the red). We used the 1 " slit width and we binned the CCD $2 \times 2$, resulting in resolving powers of 28000 in the blue and 22000 in the red. We reduced and calibrated the data using $\operatorname{IRAF}^{3}$. We carried out the observations during the nights 22 and 23 of September 2012.

The UVES (archived) observations of our objects were performed in a dichroic mode, resulting in small gaps $\sim 8 \mathrm{~nm}$ wide at $458 \mathrm{~nm}$ and $564 \mathrm{~nm}$ in the final merged spectrum (Koester et al. 2009). The resolving power at $\mathrm{H} \alpha$ is 18500. The total wavelength range covered is $\sim 350-665 \mathrm{~nm}$. We used the ESO processed (i.e. reduced plus calibrated) data. The data were obtained in service mode during 2001 and 2002 .

Combining the VLT/UVES, Gemini South/GMOS and Magellan Clay/MIKE data we count a total of 58, 25 and 22 spectra for SDSSJ0318-0107, HE0410-1137 and WD0028474 , respectively (see Table 2 ). In all cases the spectra cover the $\mathrm{H} \alpha$ line, which is used to measure the radial velocities of the white dwarf components in each of our CDWDs.

\section{ANALYSIS}

In this section we explain how we derived the radial velocities of each white dwarf component and we give details on how we measured the orbital periods and mass ratios of the three CDWDs.

\subsection{Radial velocities}

We used the double-lined $\mathrm{H} \alpha$ absorption to track the motion of the two white dwarfs in all three of our CDWDs. All the white dwarfs observed displayed sharp cores to the line that allowed velocities to be measured with high precision. Initially, we visually identified the spectra in which

3 IRAF is distributed by the National Optical Astronomy Observatories. both white dwarf components were clearly visible and separated. These spectra were then fitted with a combination of a second order polynomial (to fit the continuum and account for the very broad absorption wings seen in white dwarfs, i.e. $\sim 150-200 \mathrm{~km} / \mathrm{s}$ ) and two Gaussian components for the cores of each white dwarf. An example of these initial fits is shown in Fig. 1. We then fixed the width and strength of the Gaussians and fitted all the spectra allowing only their velocities to vary. This meant that we could reliably fit those spectra in which the two components were blended together, although spectra taken near the conjunction phases only yield a single velocity measurement for both stars.

In HE0410-1137 and WD0028-474, the two white dwarfs have different $\mathrm{H} \alpha$ profiles. This is seen best in Fig. 1. In both cases, one absorption component is much stronger than the other one, implying that we could easily associate the fitted velocities to each individual white dwarf. However, for SDSSJ0318-0107 the two white dwarfs have essentially identical line profiles. Thus, it was impossible to assign the observed spectra to individual white dwarfs for different observing runs. Therefore, since the sum of the radial velocities has a sinusoidal shape phased on the orbital period, with an amplitude given by the difference between the two white dwarf radial velocity amplitudes, for this binary system we ran a periodogram of the sum of the velocities of the two white dwarfs. We used this to determine a first estimate of the orbital period. This allowed us to identify from which white dwarf each velocity measurement came from.

The radial velocities for each white dwarf component are listed in Table 2. Hereafter we flag the white dwarf component with the deeper $\mathrm{H} \alpha$ core as white dwarf number 1 , and the white dwarf with the weaker $\mathrm{H} \alpha$ core as number 2 . In the case of SDSSJ0318-0107, where the cores have the same depth, we designate the two white dwarfs with the numbers 1 and 2 as well, but in this case the choice was arbitrary.

\subsection{Orbital periods and mass ratios}

We run ORT periodograms (Schwarzenberg-Czerny 1996) to the radial velocity data of the white dwarf component number 1 in each binary. The resulting periodograms are shown in the left panels of Fig. 2. In all cases these periodograms show a clear peak, which we interpret as the corresponding orbital periods of the binaries. The same results were obtained when running the periodograms to the white dwarf components number 2 . The radial velocities of the two white dwarfs in each binary folded over the determined orbital periods are displayed in the right panels of Fig. 2. We performed a sine fit of the form

$$
V_{\mathrm{r}}=K \sin (2 \pi \phi)+\gamma,
$$

to the folded radial velocity curves to obtain the semiamplitude velocities of the white dwarfs $K_{1}$ and $K_{2}$, where $\phi$ is the orbital phase and $\gamma$ are the systemic velocities. Once the semi-amplitude velocities were derived it became straightforward to derive the mass ratio of our CDWDs using $q=K_{1} / K_{2}$. The orbital periods, semi-amplitude velocities, systemic velocities and mass ratios are reported in Table 3 . 
Table 2. Radial velocities measured for the white dwarf components in each of our three double-lined CDWDs. We flag the white dwarf with the stronger (deeper) $\mathrm{H} \alpha$ core with 1 , the white dwarf with the weaker H $\alpha$ core with 2 . We also indicate the telescope/instrument used for obtaining the spectra from which we measured the radial velocities. The heliocentric Julian dates (HJD) in boldface correspond to the times at which the spectra shown in Fig. 1 were taken.

\begin{tabular}{|c|c|c|c|c|c|c|c|c|c|}
\hline $\begin{array}{l}\text { HJD } \\
\text { (days) }\end{array}$ & $\begin{array}{c}\mathrm{RV}_{1} \\
(\mathrm{~km} / \mathrm{s})\end{array}$ & $\begin{array}{c}\text { Error } \\
(\mathrm{km} / \mathrm{s})\end{array}$ & $\begin{array}{c}\mathrm{RV}_{2} \\
(\mathrm{~km} / \mathrm{s})\end{array}$ & $\begin{array}{c}\text { Error } \\
(\mathrm{km} / \mathrm{s})\end{array}$ & $\begin{array}{c}\text { HJD } \\
(\mathrm{km} / \mathrm{s})\end{array}$ & $\begin{array}{c}\mathrm{RV}_{1} \\
(\mathrm{~km} / \mathrm{s})\end{array}$ & $\begin{array}{c}\text { Error } \\
(\mathrm{km} / \mathrm{s})\end{array}$ & $\begin{array}{c}\mathrm{RV}_{2} \\
(\mathrm{~km} / \mathrm{s})\end{array}$ & Error \\
\hline SDSSJ0318-0107 & & & & & WD0028-474 & & & & \\
\hline VLT/UVES & & & & & VLT/UVES & & & & \\
\hline 2451947.5838 & -0.57 & 2.13 & 129.55 & 2.14 & 2452212.7188 & -33.57 & 2.79 & 128.53 & 3.80 \\
\hline 2451949.5505 & 11.46 & 3.19 & 118.46 & 3.12 & 2452272.5422 & 84.48 & 2.42 & -21.71 & 3.25 \\
\hline 2452194.6562 & 80.14 & 1.74 & 59.23 & 1.72 & 2452591.5258 & 165.00 & 4.50 & -120.61 & 6.15 \\
\hline 2452194.7051 & 95.30 & 1.60 & 50.12 & 1.58 & 2452592.5702 & -11.37 & 4.48 & 106.75 & 6.15 \\
\hline 2452194.7375 & 100.53 & 1.52 & 44.42 & 1.51 & Magellan/MIKE & & & & \\
\hline 2452194.7641 & 107.67 & 1.34 & 40.08 & 1.33 & 2456193.7920 & -75.91 & 4.66 & 193.72 & 6.02 \\
\hline 2452194.8006 & 116.61 & 1.20 & 33.98 & 1.14 & 2456193.8194 & -37.11 & 3.94 & 144.71 & 5.15 \\
\hline 2452194.8431 & 124.71 & 1.17 & 25.79 & 1.16 & 2456193.8302 & -26.15 & 3.94 & 115.79 & 5.16 \\
\hline 2452194.8763 & 129.96 & 1.16 & 21.51 & 1.14 & 2456193.8418 & -13.90 & 5.26 & 90.59 & 6.99 \\
\hline 2452195.6722 & 35.20 & 1.86 & 98.21 & 1.89 & 2456193.8817 & 70.30 & 3.85 & -18.30 & 5.39 \\
\hline 2452195.7142 & 24.10 & 1.72 & 105.86 & 1.76 & 2456193.8925 & 88.29 & 3.68 & -28.79 & 5.38 \\
\hline 2452195.7469 & 15.07 & 1.84 & 110.90 & 1.88 & Gemini/GMOS & & & & \\
\hline 2452195.7879 & 9.92 & 1.55 & 121.69 & 1.58 & 2456514.9258 & 117.92 & 3.55 & -65.75 & 4.91 \\
\hline 2452195.8179 & 0.15 & 1.25 & 124.27 & 1.26 & 2456546.7754 & -55.19 & 7.50 & 125.97 & 10.09 \\
\hline 2452195.8365 & 0.50 & 1.30 & 126.37 & 1.32 & 2456600.6630 & 142.13 & 4.10 & -114.85 & 5.61 \\
\hline 2452195.8683 & -5.18 & 1.19 & 130.06 & 1.20 & 2456601.5683 & 3.91 & 4.49 & 73.03 & 5.93 \\
\hline 2452589.6080 & 58.03 & 2.13 & 76.35 & 2.13 & 2456618.6288 & 141.43 & 4.04 & -128.92 & 5.48 \\
\hline 2452589.6732 & 43.37 & 2.30 & 89.10 & 2.27 & 2456619.6572 & -0.91 & 4.67 & 93.94 & 6.20 \\
\hline 2452589.7374 & 30.26 & 2.18 & 100.55 & 2.14 & 2456623.6102 & 99.90 & 6.96 & -55.56 & 9.75 \\
\hline 2452589.7874 & 16.43 & 2.07 & 110.12 & 2.02 & 2456626.7102 & 85.06 & 5.85 & -23.20 & 8.09 \\
\hline 2452590.6488 & 88.89 & 2.25 & 53.50 & 2.25 & 2456627.6687 & 64.77 & 4.47 & -1.58 & 6.11 \\
\hline 2452590.7623 & 115.39 & 2.13 & 31.98 & 2.24 & 2456628.6470 & 62.80 & 5.66 & -12.38 & 7.90 \\
\hline 2452590.8231 & 128.62 & 2.60 & 20.35 & 2.77 & 2456630.5878 & 63.84 & 5.68 & -8.46 & 7.91 \\
\hline 2452591.5618 & 51.40 & 2.55 & 87.64 & 2.53 & 2456639.5505 & 65.88 & 6.89 & -14.66 & 9.58 \\
\hline 2452591.5987 & 40.73 & 2.64 & 93.77 & 2.58 & & & & & \\
\hline 2452591.6458 & 29.79 & 2.53 & 99.64 & 2.45 & HE0410-1137 & & & & \\
\hline 2452591.7048 & 20.62 & 2.25 & 113.29 & 2.18 & VLT/UVES & & & & \\
\hline 2452591.7555 & 6.073 & 2.30 & 118.38 & 2.24 & 2452334.5615 & 10.37 & 1.30 & 155.61 & 2.33 \\
\hline 2452591.8297 & -6.797 & 2.83 & 131.98 & 2.74 & 2452338.5410 & 45.95 & 1.51 & 91.07 & 2.51 \\
\hline 2452592.5638 & 90.12 & 2.96 & 55.94 & 2.97 & 2452589.6632 & 139.16 & 1.95 & -25.16 & 3.88 \\
\hline 2452592.6148 & 104.73 & 5.01 & 40.49 & 4.96 & 2452589.7183 & 130.14 & 2.09 & -9.91 & 5.09 \\
\hline 2452592.6629 & 114.80 & 3.07 & 35.90 & 3.14 & 2452589.7764 & 92.19 & 1.58 & 44.83 & 2.77 \\
\hline 2452592.6965 & 123.36 & 2.65 & 29.08 & 2.72 & 2452589.8418 & 39.73 & 1.90 & 108.69 & 3.57 \\
\hline 2452592.7523 & 131.75 & 2.66 & 16.20 & 2.81 & 2452590.6810 & 143.39 & 2.00 & -25.96 & 5.47 \\
\hline 2452592.7968 & 141.09 & 2.62 & 12.71 & 2.73 & 2452590.7722 & 105.15 & 1.86 & 34.95 & 3.97 \\
\hline 2452592.8334 & 145.44 & 2.48 & 7.17 & 2.62 & 2452590.8333 & 64.35 & 3.22 & 64.35 & 3.22 \\
\hline Magellan/MIKE & & & & & 2452591.5886 & 73.98 & 3.53 & 73.98 & 3.53 \\
\hline 2456192.7039 & 129.92 & 2.71 & 23.62 & 2.85 & 2452591.6355 & 116.21 & 2.36 & 12.26 & 4.21 \\
\hline 2456192.7286 & 132.31 & 2.65 & 15.59 & 2.76 & 2452592.5842 & 64.64 & 3.08 & 64.64 & 3.08 \\
\hline 2456192.7407 & 135.36 & 2.70 & 8.24 & 2.85 & 2452592.7420 & 139.02 & 2.88 & -24.88 & 6.86 \\
\hline 2456192.7657 & 139.97 & 2.66 & 9.83 & 2.76 & 2452592.8435 & 70.23 & 3.07 & 70.23 & 3.07 \\
\hline 2456192.8038 & 146.19 & 2.44 & 8.78 & 2.64 & Magellan/MIKE & & & & \\
\hline 2456192.8652 & 150.59 & 3.13 & 0.31 & 3.39 & 2456192.7897 & 99.59 & 1.79 & 38.77 & 2.04 \\
\hline 2456193.7152 & -7.21 & 2.28 & 126.34 & 2.15 & 2456192.8294 & 66.93 & 3.07 & 66.93 & 3.07 \\
\hline 2456193.8550 & -19.61 & 2.17 & 139.15 & 2.07 & 2456192.8409 & 54.61 & 1.68 & 106.9 & 4.02 \\
\hline Gemini/GMOS & & & & & 2456192.8517 & 51.89 & 1.57 & 112.3 & 2.71 \\
\hline 2455818.7358 & 5.83 & 0.92 & 113.99 & 0.92 & 2456193.7473 & 133.46 & 1.49 & -9.52 & 3.84 \\
\hline 2455820.7834 & 10.39 & 3.14 & 124.54 & 3.08 & 2456193.7769 & 126.19 & 2.04 & 126.19 & 2.04 \\
\hline 2455820.7802 & -3.73 & 0.95 & 130.32 & 0.94 & 2456193.8040 & 101.54 & 2.12 & 20.89 & 4.34 \\
\hline 2456514.9099 & 32.18 & 2.49 & 102.33 & 2.38 & 2456193.8662 & 43.89 & 1.70 & 96.04 & 2.64 \\
\hline 2456515.8935 & 123.51 & 2.45 & 46.08 & 2.56 & Gemini/GMOS & & & & \\
\hline 2456545.8853 & -16.03 & 3.57 & 122.59 & 3.41 & 2456515.9078 & -0.03 & 12.21 & 119.39 & 10.90 \\
\hline 2456599.7406 & 39.31 & 3.24 & 104.96 & 3.23 & 2456600.7618 & 102.36 & 2.62 & 21.77 & 2.86 \\
\hline 2456600.7452 & 95.24 & 3.82 & 46.63 & 3.86 & 2456627.5403 & 108.99 & 2.21 & 40.10 & 3.97 \\
\hline 2456618.6466 & -5.27 & 2.98 & 136.72 & 2.90 & & & & & \\
\hline 2456619.6750 & 135.92 & 2.80 & 23.74 & 2.99 & & & & & \\
\hline 2456626.7269 & 91.66 & 2.82 & 46.36 & 2.83 & & & & & \\
\hline 2456627.6865 & 39.09 & 2.92 & 88.47 & 2.85 & & & & & \\
\hline 2456630.6417 & 103.57 & 4.75 & 44.78 & 5.02 & & & & & \\
\hline 2456639.5702 & -1.05 & 4.00 & 142.62 & 3.90 & & & & MNRAs & $000,1-$ \\
\hline
\end{tabular}



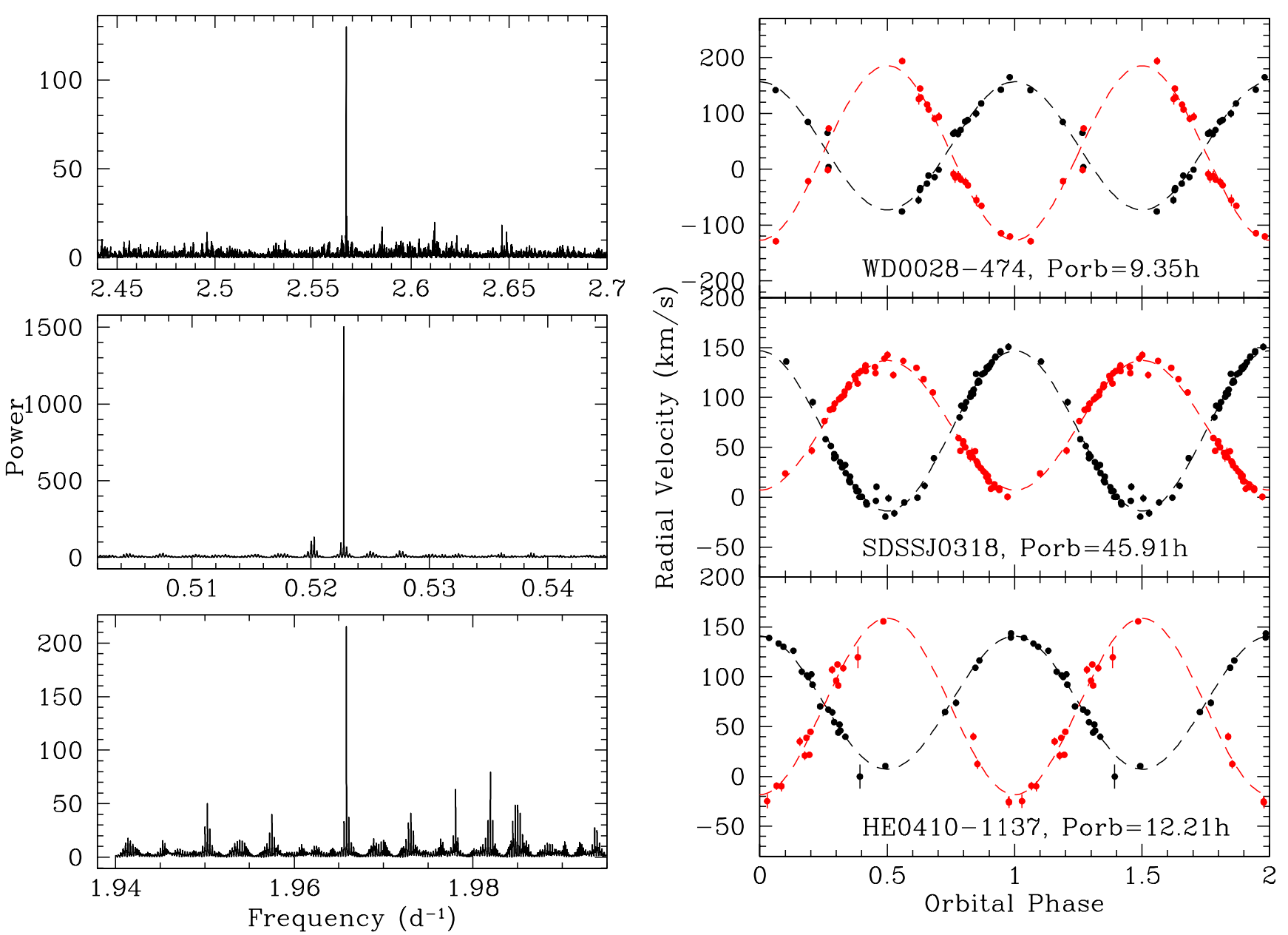

Figure 2. Left panels: ORT periodograms indicating the orbital periods of our three CDWDs. Right panels: phase-folded radial velocity curves. The radial velocities measured from the white dwarf component with the deeper $\mathrm{H} \alpha$ core are shown in black, the radial velocities measured from the weaker core component in red. Solid dots indicate radial velocities measured from the VLT/UVES spectra, open circles from the Magelan/MIKE spectra and stars from the Gemini/GMOS spectra. The radial velocity errors are in most cases smaller than the symbol sizes. The red and black dashed lines represent the best fits to the radial velocities.

Table 3. Stellar and orbital parameters of the three CDWD studied in this work. In order of appearance are the orbital period, the semi-amplitude velocities, the $\gamma$ systemic velocities, the mass ratio, the masses and the effective temperatures.

\begin{tabular}{cccc}
\hline \hline & WD0028-474 & SDSSJ0318-0107 & HE0410-1137 \\
\hline$P_{\text {orb }}(\mathrm{h})=$ & $9.350 \pm 0.007$ & $45.908 \pm 0.006$ & $12.208 \pm 0.008$ \\
$\gamma_{1}(\mathrm{~km} / \mathrm{s})=$ & $41.8 \pm 1.0$ & $66.4 \pm 0.3$ & $74.0 \pm 0.6$ \\
$\gamma_{2}(\mathrm{~km} / \mathrm{s})=$ & $29.1 \pm 1.3$ & $72.1 \pm 0.3$ & $70.1 \pm 1.2$ \\
$K_{1}(\mathrm{~km} / \mathrm{s})=$ & $114.8 \pm 1.6$ & $80.2 \pm 0.5$ & $66.7 \pm 0.7$ \\
$K_{2}(\mathrm{~km} / \mathrm{s})=$ & $156.1 \pm 2.2$ & $65.1 \pm 0.6$ & $88.4 \pm 1.3$ \\
$q=$ & $0.735 \pm 0.014$ & $1.233 \pm 0.013$ & $0.755 \pm 0.014$ \\
$M_{1}\left(\mathrm{M}_{\odot}\right)=$ & $0.60 \pm 0.06$ & $0.40 \pm 0.05$ & $0.51 \pm 0.04$ \\
$M_{2}\left(\mathrm{M}_{\odot}\right)=$ & $0.45 \pm 0.04$ & $0.49 \pm 0.05$ & $0.39 \pm 0.03$ \\
$T_{1}(\mathrm{~K})=$ & $18500 \pm 500$ & $14500 \pm 500$ & $16000 \pm 500$ \\
$T_{2}(\mathrm{~K})=$ & $17000 \pm 500$ & $13500 \pm 500$ & $19000 \pm 500$ \\
\hline
\end{tabular}

\section{MASSES}

In this section we describe the method employed to measure the component masses of the white dwarfs of each binary. These were obtained applying three independent observational constraints. The first and most obvious is that the mass components need to comply with the measured mass ratios - see Sect. 3.2. The second constraint comes from the $\mathrm{H} \alpha$ core ratio, i.e. the flux ratio between the depth of the $\mathrm{H} \alpha$ cores arising from the two white dwarf components. We obtained this ratio directly from the observed double-lined spectra - see Fig. 1 and also Sect. 2. For each of our three CDWDs we measured the $\mathrm{H} \alpha$ core ratio from all available individual spectra, where we determined the flux of each core as the minimum flux of the considered absorption line. We averaged the $\mathrm{H} \alpha$ core ratios derived from all available spectra, which were found to be nearly identical for each CDWD. We obtained the third constraint by model-fitting the observed CDWD spectra corrected from the orbital motion. More details are given below.

We used a set of 612 white dwarf model spectra from 

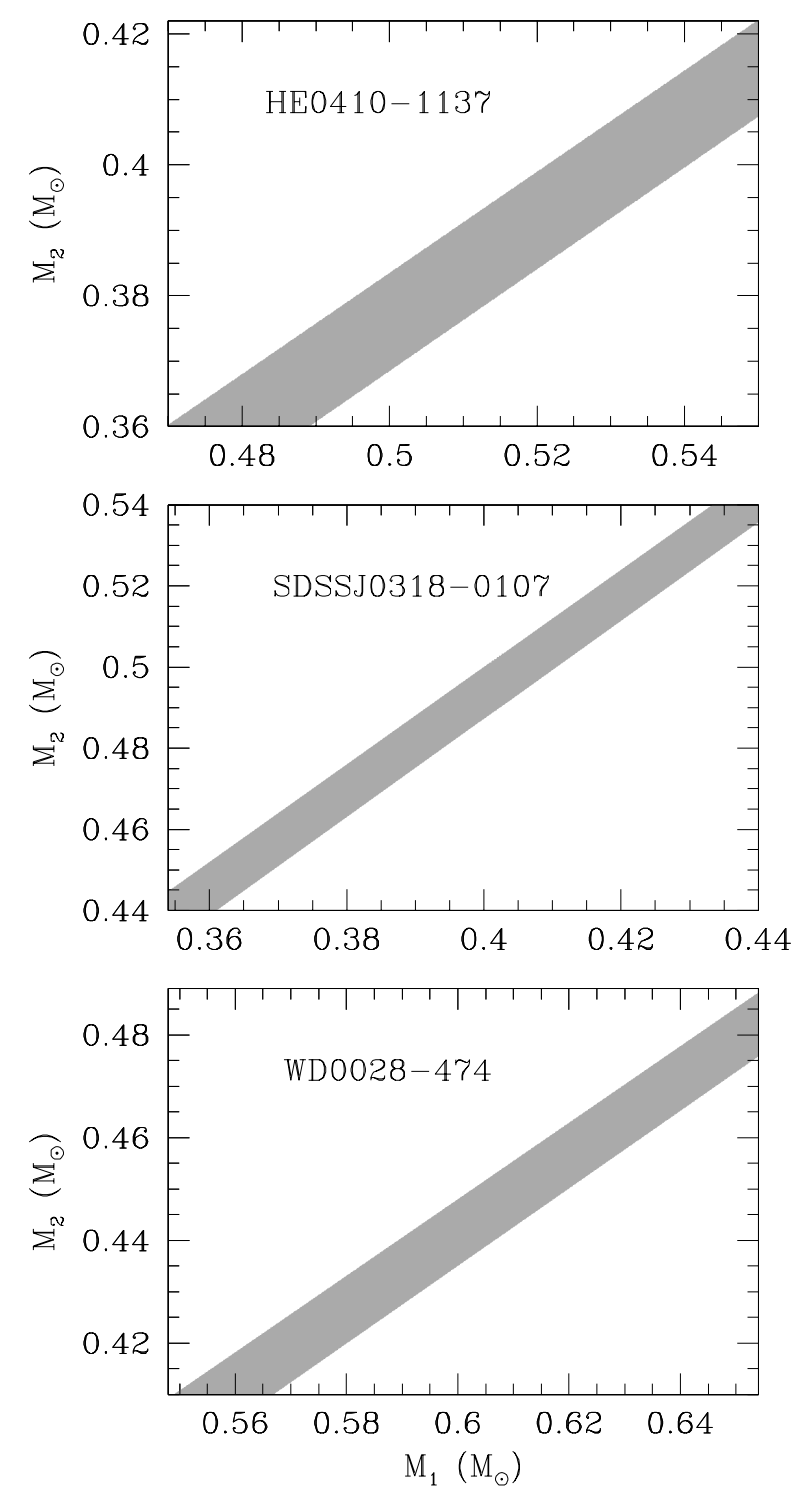

Figure 3. Mass ranges (gray shade areas) derived for the white dwarf components of the three CDWDs studied in this work. The mass ranges comply with the mass ratio $q$ obtained from the measured radial velocity semi-amplitudes (see the right panel of Fig. 2).

Koester (2010) and summed the flux of each of them with the flux of each of the remaining spectra so that we obtained model spectra of 374544 double white dwarfs (or 187272 if duplicates are discarded). The 612 model spectra included effective temperatures ranging from $6,000 \mathrm{~K}$ to $10,000 \mathrm{~K}$ in steps of $250 \mathrm{~K}$, from $10,000 \mathrm{~K}$ to $30,000 \mathrm{~K}$ in steps of $1,000 \mathrm{~K}$, from $30,000 \mathrm{~K}$ to $70,000 \mathrm{~K}$ in steps of $5,000 \mathrm{~K}$ and $70,000 \mathrm{~K}$ to $100,000 \mathrm{~K}$ in steps of $10,000 \mathrm{~K}$, and surface gravities ranging between 6.5 and 9.5 dex for each effective temperature. From each model we derived the $\mathrm{H} \alpha$ core ratio in the same way as described above. We then used the fitting routine of Rebassa-Mansergas et al. (2007) to fit the 374,544 spectra with the set of 612 models and derived effective temperatures and surface gravities for each of them. The synthetic double white dwarf spectra are the combined fluxes of two white dwarfs, hence the fitted paramater values are not representative of either white dwarf component. Consequently, we will refer to them as "combined" values. These combined values can be compared to those obtained fitting the observed spectra of our three CDWDs. To that end, we fitted all available UVES spectra of resolving power 18500 (the highest resolution among our data, see Sect. 2) corrected from orbital motion of each CDWD. The resulting fits were found to agree within the errors in the three cases and we averaged the results to obtain the final combined values of effective temperature and surface gravity from the observed spectra.

The procedure outlined above allowed us to build a grid including $\mathrm{H} \alpha$ core ratios, mass ratios and combined effective temperatures and surface gravities for each of the 374,544 double white dwarf model spectra. Given that each synthetic double white dwarf spectrum results from adding the fluxes of two individual white dwarf model spectra of known effective temperatures and surface gravities, the grid includes also these individual parameters for each white dwarf. We can easily derive masses from the effective temperature and surface gravities using white dwarf cooling sequences, hence we are also able to include the individual white dwarf component masses (hence mass ratios) for each double white dwarf model in the grid. The masses were obtained using the cooling sequences of Renedo et al. (2010) for carbonoxygen white dwarfs $\left(M_{\mathrm{WD}}\right.$ between 0.45 and $\left.1.1 M_{\odot}\right)$, Althaus et al. (2005); Althaus et al. (2007) for oxygen-neon white dwarfs $\left(M_{\mathrm{WD}}>1.1 M_{\odot}\right)$ and Serenelli et al. (2001) for helium core white dwarfs $\left(M_{\mathrm{WD}}<0.45 M_{\odot}\right)$.

For each CDWD we used the derived mass ratio, the combined fitted effective temperatures and surface gravities from the observed UVES spectra and the measured $\mathrm{H} \alpha$ core ratio to select all double white dwarf models satisfying these conditions within the grid. From the models that survived these cuts we obtained the mass and effective temperature ranges for each white dwarf component. The resulting white dwarf mass ranges obtained in this way are shown in Fig. 3 for our three CDWDs. The masses and effective temperature values are indicated in Table 3 . Inspection of Table 3 reveals that the more massive white dwarf in WD0028-474 is the hotter (younger) one. For this to be the case Moran et al. (1997) claim that such systems (we quote literally) "must have undergone a period of conservative mass transfer, during which the initial mass ratio was reversed, so that the more evolved star became the less massive, and produced the less massive white dwarf".

\section{GRAVITATIONAL REDSHIFTS}

We can use the difference in systemic velocities of the two white dwarfs, $\gamma_{1}-\gamma_{2}$, as a consistency check of our derived masses. This is because the difference in systemic velocities is related to the difference in gravitational redshifts of the two white dwarfs. The gravitational redshift of white dwarfs (in $\mathrm{km} / \mathrm{s}$ ) is given by

$$
Z=0.635\left(\frac{M}{R}\right),
$$

where the mass and radius are expressed in solar units (Koester 1987). In a close binary composed of two white 
dwarfs the above equation becomes

$$
Z_{1}=0.635\left(\frac{M_{1}}{R_{1}}+\frac{M_{2}}{a}\right),
$$

where $a$ is the orbital separation, also given in solar radii. This expression takes into account the gravitational potential acting on the white dwarf owing to the other white dwarf (we have not included the effects caused by the difference in transverse Doppler shifts, since these are negligible in these binaries). Therefore, $\Delta Z=Z_{1}-Z_{2}$ should be equal to the difference in systemic velocities $\gamma_{1}-\gamma_{2}$.

Adopting the masses listed in Table 3, using Kepler's third law to derive the orbital separations, and employing the mass-radius relation of Renedo et al. (2010) we find $\Delta Z=11.7 \pm 3.0 \mathrm{~km} / \mathrm{s}$ for WD0028-474, $\Delta Z=7.1 \pm$ $2.7 \mathrm{~km} / \mathrm{s}$ for SDSSJ0318-0107 and $\Delta Z=5.3 \pm 1.8 \mathrm{~km} / \mathrm{s}$ for HE0410-1137. These values are in excellent agreement with the difference in systemic velocities: $12.7 \pm 1.6 \mathrm{~km} / \mathrm{s}$ for WD0028-474, $5.7 \pm 0.4 \mathrm{~km} / \mathrm{s}$ for SDSSJ318-0107 and $3.9 \pm 1.3 \mathrm{~km} / \mathrm{s}$ for HE0410-1137. This result indicates that our method for deriving the component masses of the three CDWDs studied in this work is reliable.

\section{MERGER TIMES}

The orbital separation of a CDWD decreases in time due to the emission of gravitational waves until it eventually merges. The time in Myr needed for such an event is given by

$$
\tau=47925 \frac{\left(M_{1}+M_{2}\right)^{1 / 3}}{M_{1} M_{2}} P^{8 / 3}
$$

where $P$ is the orbital period in days and the masses are in units of $\mathrm{M}_{\odot}$ (Kraft et al. 1962). Here we make use of the orbital periods and component masses determined in the previous sections to estimate when our three CDWDs will merge. We obtain 14.6, 1326 and 38.4 Gyr for WD0028-474, SDSSJ0318-0107 and HE0410-1137, respectively. Thus, in all cases more than the Hubble time is needed to merge. The combined masses of the three CDWDs do not exceed the Chandrasekhar mass. Hence, they are expected to become single massive $\left(\gtrsim 0.9 \mathrm{M}_{\odot}\right)$ white dwarfs.

\section{CONCLUSIONS}

We have presented intense follow-up spectroscopy of three double-lined binary white dwarfs. Analysing their spectra we have been able to derive precise values of the orbital periods and mass ratios. We have also derived the white dwarf component masses and effective temperatures of each binary applying a new method based on mass ratio, $\mathrm{H} \alpha$ core ratio and spectral model-fitting constraints. The three systems will need more than the Hubble time to merge. After this they are expected to become single massive $\left(\gtrsim 0.9 \mathrm{M}_{\odot}\right)$ white dwarfs.

Our work increases the number of double white dwarfs with available orbital periods and component masses by $\sim 20$ per cent, to a total of 21 systems. Reconstructing the evolution of these objects is expected to dramatically help in providing the much needed new insights into the formation of close double white dwarfs, which is also essential to predict the rates of Type Ia supernovae produced by the doubledegenerate channel (van der Sluys et al. 2006; Woods et al. 2012).

\section{ACKNOWLEDGMENTS}

This research has been funded by MINECO grant AYA201459084-P, by the AGAUR, by the European Research Council under the European Union's Seventh Framework Programme (FP/2007-2013)/ERC Grant Agreement n.320964 (WDTracer), by Milenium Science Initiative, Chilean Ministry of Economy, Nucleus P10-022-F and by Fondecyt $(1100782,3140585)$. We thank the anonymous referee for his/her suggestions and Tom Marsh for the use of the molly software. ARM acknowledges helpful discussions with Elme Breedt.

Based on observations performed with the Gemini South Telescope (program GS-2013B-Q-23) and the Magellan Clay Telescope. Based on data products from observations made with ESO Telescopes at the La Silla Paranal Observatory under programmes 167.D-0407(A) and 70.D0334(A), PI R. Napiwotzki.

\section{REFERENCES}

Althaus, L. G., García-Berro, E., Isern, J., Córsico, A. H., 2005, A\&A, 441, 689

Althaus, L. G., García-Berro, E., Isern, J., Córsico, A. H., Rohrmann, R. D., 2007, AA, 465, 249

Badenes, C., Maoz, D., 2012, ApJ, 749, L11

Bergeron, P., Liebert, J., 2002, ApJ, 566, 1091

Bours, M. C. P., et al., 2014, MNRAS, 438, 3399

Brown, W. R., Kilic, M., Hermes, J. J., Allende Prieto, C., Kenyon, S. J., Winget, D. E., 2011, ApJ, 737, L23

Di Stefano, R., 2010, ApJ, 712, 728

Hallakoun, N., et al., 2016, MNRAS, 458, 845

Hils, D., Bender, P. L., Webbink, R. F., 1990, ApJ, 360, 75

Iben, I. J., Livio, M., 1993, PASP, 105, 1373

Kaplan, D. L., et al., 2014, ApJ, 780, 167

Karl, C., Napiwotzki, R., Heber, U., Lisker, T., Nelemans, G., Christlieb, N., Reimers, D., 2003a, in de Martino, D., Silvotti, R., Solheim, J.-E., Kalytis, R., eds., NATO ASIB Proc. 105: White Dwarfs, vol. 105, p. 43

Karl, C. A., Napiwotzki, R., Nelemans, G., Christlieb, N., Koester, D., Heber, U., Reimers, D., 2003b, A\&A, 410, 663

Kilic, M., et al., 2011, MNRAS, 413, L101

Kilic, M., et al., 2014, MNRAS, 438, L26

Koester, D., 1987, ApJ, 322, 852

Koester, D., 2010, Mem. Soc. Astron. Italiana, 81, 921

Koester, D., Kepler, S. O., Kleinman, S. J., Nitta, A., 2009, Journal of Physics Conference Series, 172, 012006

Kraft, R. P., Mathews, J., Greenstein, J. L., 1962, ApJ, 136, 312

Maoz, D., Hallakoun, N., 2016, ArXiv e-prints

Marsh, T. R., 1995, MNRAS, 275, L1

Marsh, T. R., 2011, Classical and Quantum Gravity, 28, 094019

Marsh, T. R., Gänsicke, B. T., Steeghs, D., Southworth, J., Koester, D., Harris, V., Merry, L., 2011, ApJ, 736, 95

Maxted, P. F. L., Marsh, T. R., Moran, C. K. J., Han, Z., 2000, MNRAS, 314, 334

Maxted, P. F. L., Burleigh, M. R., Marsh, T. R., Bannister, N. P., 2002a, MNRAS, 334, 833

Maxted, P. F. L., Marsh, T. R., Moran, C. K. J., 2002b, MNRAS, 332,745

Moran, C., Marsh, T. R., Bragaglia, A., 1997, MNRAS, 288, 538 
Napiwotzki, R., et al., 2002, A\&A, 386, 957

Napiwotzki, R., et al., 2007, in R. Napiwotzki \& M. R. Burleigh, ed., 15th European Workshop on White Dwarfs, vol. 372 of Astronomical Society of the Pacific Conference Series, p. 387

Nelemans, G., Tout, C. A., 2005, MNRAS, 356, 753

Nelemans, G., Verbunt, F., Yungelson, L. R., Portegies Zwart, S. F., 2000, A\&A, 360, 1011

Parsons, S. G., Marsh, T. R., Gänsicke, B. T., Drake, A. J., Koester, D., 2011, ApJ, 735, L30

Rebassa-Mansergas, A., Gänsicke, B. T., Rodríguez-Gil, P., Schreiber, M. R., Koester, D., 2007, MNRAS, 382, 1377

Rebassa-Mansergas, A., Rybicka, M., Liu, X.-W., Han, Z., GarcíaBerro, E., 2015, MNRAS, 452, 1637

Renedo, I., Althaus, L. G., Miller Bertolami, M. M., Romero, A. D., Córsico, A. H., Rohrmann, R. D., García-Berro, E., 2010, ApJ, 717, 183

Ruiter, A. J., Belczynski, K., Benacquista, M., Larson, S. L., Williams, G., 2010, ApJ, 717, 1006

Saffer, R. A., Liebert, J., Olszewski, E. W., 1988, ApJ, 334, 947

Schwarzenberg-Czerny, A., 1996, ApJ, 460, L107

Serenelli, A. M., Althaus, L. G., Rohrmann, R. D., Benvenuto, O. G., 2001, MNRAS, 325, 607

Steinfadt, J. D. R., Kaplan, D. L., Shporer, A., Bildsten, L., Howell, S. B., 2010, ApJ, 716, L146

Toonen, S., Nelemans, G., Portegies Zwart, S., 2012, A\&A, 546, A70

van der Sluys, M. V., Verbunt, F., Pols, O. R., 2006, A\&A, 460, 209

Vennes, S., et al., 2011, ApJ, 737, L16

Webbink, R. F., 1984, ApJ, 277, 355

Webbink, R. F., 2008, in E. F. Milone, D. A. Leahy, \& D. W. Hobill, ed., Astrophysics and Space Science Library, vol. 352 of Astrophysics and Space Science Library, p. 233

Woods, T. E., Ivanova, N., van der Sluys, M. V., Chaichenets, S., 2012, ApJ, 744, 12

Zuo, Z.-Y., Li, X.-D., 2014, MNRAS, 442, 1980 